\title{
Exact multiplicity of solutions for discrete second order Neumann boundary value problems
}

Dingyong Bai ${ }^{1,2}$, Hairong Lian ${ }^{3}$ and Haiyan Wang ${ }^{4,5,6^{*}}$

\section{"Correspondence:}

Haiyan.Wang@asu.edu

${ }^{4}$ Department of Mathematics,

Northwest Normal University,

Lanzhou, 730070, P.R. China

${ }^{5}$ Department of Mathematics,

North University of China, Taiyuan,

Shanxi 030051, P.R. China

Full list of author information is available at the end of the article

\begin{abstract}
Our concern is the second order difference equation $\Delta^{2} u(t-1)+g(u(t))=h(t)$ subject to the Neumann boundary conditions $\Delta u(0)=\Delta u(T)=0$. Under convex/concave conditions imposed on $g$, some results on the exact numbers of solutions and positive solutions are established based on the discussions to the maximum and minimum numbers of (positive) solutions.
\end{abstract}

Keywords: difference equation; Neumann boundary value problem; exact numbers of solutions and positive solutions

\section{Introduction}

For $a, b \in \mathbf{Z}$ with $a<b$, define $[a, b]_{\mathbf{Z}}=\{a, a+1, a+2, \ldots, b-1, b\}$. Consider the following Neumann boundary value problem:

$$
\left\{\begin{array}{l}
\Delta^{2} u(t-1)+g(u(t))=h(t), \quad t \in[1, T]_{\mathrm{Z}} \\
\Delta u(0)=\Delta u(T)=0
\end{array}\right.
$$

where $h:[1, T]_{\mathbf{Z}} \rightarrow \mathbf{R}, \Delta u(t)=u(t+1)-u(t)$ and $T>3$ is a given positive integer. Our purpose is to find the exact number of solutions and positive solutions of (1.1).

In these last years, the existence and multiplicity of solutions for nonlinear discrete problems subject to various boundary value conditions have been widely studied by using different abstract methods such as critical point theory, fixed point theorems, lower and upper solutions method, and Brower degree (see, e.g., [1-17] and the references therein). All these results are about the unique solution, or the minimum amount of solutions, and positive solutions. To the best of our knowledge, there is no report on the exact number of solutions for discrete boundary value problems.

For BVPs of differential equations, there are many papers concerned with the bifurcation values and exact multiplicities of solutions and positive solutions by bifurcation theory, quadrature method, time-map analysis and otherwise. See [18-29] and the references therein. For difference equations, however, the loss of continuity puts some methods used well in differential equations, such as the quadrature method and its time-map analysis, out of action. Therefore, it is very meaningful to study the exact number of solutions for 
discrete boundary value problems. In this paper, based on the discussions to the maximum and minimum numbers of (positive) solutions, we establish some results on the exact number of (positive) solutions of (1.1) under convex/concave conditions.

The remaining part of this paper is organized as follows. In Section 2, under the Neumann boundary conditions $\Delta u(0)=\Delta u(T)=0$, we show the Green's functions of the linear difference operators $-\Delta^{2} u(t-1)+L u(t)$ and $\Delta^{2} u(t-1)+K u(t)$, where $L$ and $K$ are two positive constants with $K<4 \sin ^{2} \frac{\pi}{2 T}$. Then, in Section 3, we make some estimates on the maximum number of solutions of (1.1), and, in Section 4, we establish some results on the minimum numbers of solutions and positive solutions of (1.1). Finally, we give the results on the exact multiplicities of solutions and positive solutions of (1.1) in Section 5.

\section{Green's functions}

In this section, we show the Green's functions of the linear difference operators $-\Delta^{2} u(t-$ $1)+L u(t)$ and $\Delta^{2} u(t-1)+K u(t)$ satisfying the Neumann boundary conditions $\Delta u(0)=$ $\Delta u(T)=0$.

Let $0<K<4 \sin ^{2} \frac{\pi}{2 T}$. Consider the Neumann boundary value problem

$$
\left\{\begin{array}{l}
\Delta^{2} u(t-1)+K u(t)=h(t), \quad t \in[1, T]_{\mathrm{Z}} \\
\Delta u(0)=\Delta u(T)=0
\end{array}\right.
$$

It is easy to see that the corresponding homogeneous equation $\Delta^{2} u(t-1)+K u(t)=0$ has two independent real solutions $\cos \theta t$ and $\sin \theta t$, where $\theta$ is defined by

$$
\theta=\arccos \left(1-\frac{K}{2}\right), \quad 0<\theta<\frac{\pi}{T} .
$$

Lemma 2.1 Problem (2.1) has a unique solution

$$
u(t)=\sum_{s=1}^{T} G_{1}(t, s) h(s)
$$

where

$$
G_{1}(t, s)=\frac{1}{\sin \theta \sin \theta T} \begin{cases}\cos \frac{2 s-1}{2} \theta \cos \frac{2 T+1-2 t}{2} \theta, & 1 \leq s \leq t \leq T+1, \\ \cos \frac{2 t-1}{2} \theta \cos \frac{2 T+1-2 s}{2} \theta, & 0 \leq t \leq s \leq T\end{cases}
$$

In addition, if $0<K<4 \sin ^{2} \frac{\pi}{4 T}$, then $G_{1}(t, s)>0$ for all $(t, s) \in[0, T+1]_{\mathrm{Z}} \times[1, T]_{\mathrm{Z}}$.

Proof First, we show that $u$ given by (2.3) solves (2.1). By $G_{1}(0, s)=G_{1}(1, s)$ we know that $u(0)=u(1)$, i.e., $\Delta u(0)=0$. Similarly, $G_{1}(T, s)=G_{1}(T+1, s)$ implies that $\Delta u(T)=0$. Now we show that $u$ satisfies the equation $\Delta^{2} u(t-1)+K u(t)=h(t)$, i.e., $u(t+1)+(K-2) u(t)+$ $u(t-1)=h(t), t \in[1, T]_{\mathrm{Z}}$. Since

$$
\begin{aligned}
& u(t+1)+(K-2) u(t)+u(t-1) \\
& \quad=\sum_{s=1}^{T}\left[G_{1}(t+1, s)+(K-2) G_{1}(t, s)+G_{1}(t-1, s)\right] h(s)
\end{aligned}
$$




$$
\begin{aligned}
= & \left(\sum_{s=1}^{t-1}+\sum_{s=t+1}^{T}\right)\left[G_{1}(t+1, s)+(K-2) G_{1}(t, s)+G_{1}(t-1, s)\right] h(s) \\
& +\left[G_{1}(t+1, t)+(K-2) G_{1}(t, t)+G_{1}(t-1, t)\right] h(t) \\
:= & \sum_{s=1}^{t-1} I_{1}(t, s) h(s)+\sum_{s=t+1}^{T} I_{2}(t, s) h(s)+I_{3}(t) h(t)
\end{aligned}
$$

we just need to verify that $I_{1} \equiv 0, I_{2} \equiv 0$, and $I_{3} \equiv 1$. In fact, by the fact that $\cos \theta t$ solves the homogeneous equation $\Delta^{2} u(t-1)+K u(t)=0$, we know that, for all $t \in[1, T]_{\mathrm{Z}}$ and $1 \leq s \leq t-1$,

$$
\begin{aligned}
& I_{1}(t, s) \sin \theta \sin \theta T \\
& =\cos \frac{(2 s-1) \theta}{2}\left[\cos \frac{(2 T-2 t-1) \theta}{2}+(K-2) \cos \frac{(2 T+1-2 t) \theta}{2}\right. \\
& \left.\quad+\cos \frac{(2 T+3-2 t) \theta}{2}\right] \\
& =0 .
\end{aligned}
$$

This implies $I_{1} \equiv 0$. Similarly $I_{2} \equiv 0$. Finally,

$$
\begin{aligned}
& I_{3}(t) \sin \theta \sin \theta T \\
& \quad=\cos \frac{(2(t-1)-1) \theta}{2} \cos \frac{(2 T+1-2 t) \theta}{2}-\cos \frac{(2 t-1) \theta}{2} \cos \frac{(2 T+1-2(t-1)) \theta}{2} \\
& \quad=\frac{1}{2}[\cos (T-1) \theta-\cos (T+1) \theta] \\
& =\sin \theta \sin \theta T
\end{aligned}
$$

holds for all $t \in[1, T]_{\mathrm{Z}}$, which implies $I_{3} \equiv 1$.

Since the corresponding homogeneous problem has only the trivial zero solution, problem (2.1) has a unique solution $u$, which is given by (2.1). Clearly, if $0<K<4 \sin ^{2} \frac{\pi}{4 T}$, then $0<\theta<\frac{\pi}{2 T}$ and hence $G_{1}(t, s)>0$ for all $(t, s) \in[0, T+1]_{\mathbf{Z}} \times[1, T]_{\mathbf{Z}}$. The proof is complete.

Let $L>0$. Consider the Neumann boundary value problem

$$
\left\{\begin{array}{l}
-\Delta^{2} u(t-1)+L u(t)=h(t), \quad t \in[1, T]_{\mathrm{Z}} \\
\Delta u(0)=\Delta u(T)=0
\end{array}\right.
$$

For convenience, let

$$
A=\frac{1}{2}\left(L+2+\sqrt{L^{2}+4 L}\right), \quad \rho=\left(A^{T}-A^{-T}\right)\left(A^{2}-1\right) .
$$

\section{Lemma 2.2 Problem (2.4) has a unique solution}

$$
u(t)=\sum_{s=1}^{T} G_{2}(t, s) h(s)
$$


where

$$
G_{2}(t, s)=\frac{1}{\rho} \begin{cases}\left(A^{s}+A^{-s+1}\right)\left(A^{t-T}+A^{T-t+1}\right), & 1 \leq s \leq t \leq T+1 \\ \left(A^{t}+A^{-t+1}\right)\left(A^{s-T}+A^{T-s+1}\right), & 0 \leq t \leq s \leq T\end{cases}
$$

and $G_{2}(t, s)>0$ for all $(t, s) \in[0, T+1]_{\mathbf{Z}} \times[1, T]_{\mathbf{Z}}$.

Proof First, we show that $u$ given by (2.5) solves (2.4). By $G_{2}(0, s)=G_{2}(1, s)$ we know $\Delta u(0)=0$ and by $G_{2}(T, s)=G_{2}(T+1, s), \Delta u(T)=0$. Now we show that $u$ satisfies the equation $-\Delta^{2} u(t-1)+L u(t)=h(t)$, i.e., $u(t+1)-(L+2) u(t)+u(t-1)=-h(t), t \in[1, T]_{\mathbf{Z}}$. Since

$$
\begin{aligned}
u(t+1)-(L+2) u(t)+u(t-1) \\
=\sum_{s=1}^{T}\left[G_{2}(t+1, s)-(L+2) G_{2}(t, s)+G_{2}(t-1, s)\right] h(s) \\
=\left(\sum_{s=1}^{t-1}+\sum_{s=t+1}^{T}\right)\left[G_{2}(t+1, s)-(L+2) G_{2}(t, s)+G_{2}(t-1, s)\right] h(s) \\
\quad+\left[G_{2}(t+1, t)-(L+2) G_{2}(t, t)+G_{2}(t-1, t)\right] h(t) \\
:=\sum_{s=1}^{t-1} I_{1}(t, s) h(s)+\sum_{s=t+1}^{T} I_{2}(t, s) h(s)+I_{3}(t) h(t),
\end{aligned}
$$

we just need to verify that $I_{1} \equiv 0, I_{2} \equiv 0$, and $I_{3} \equiv-1$. In fact, by the fact that $A$ is a real root of the equation $\ell(\lambda):=\lambda^{2}-(L+2) \lambda+1=0$, we know that, for all $t \in[1, T]_{\mathrm{Z}}$ and $1 \leq s \leq t-1$,

$$
\rho I_{1}(t, s)=\left(A^{s}+A^{-s+1}\right)\left(A^{t-T-1}+A^{T-t}\right) \ell(A)=0,
$$

holds, which implies $I_{1} \equiv 0$. Similarly $I_{2} \equiv 0$. For $I_{3}$, we have, for all $t \in[1, T]_{\mathrm{Z}}$,

$$
\begin{aligned}
\rho I_{3}(t)= & \left(A^{t}+A^{-t+1}\right)\left[A^{t-T-1}\left(A^{2}-(L+2) A\right)+A^{T-t}(1-(L+2) A)\right] \\
& +\left(A^{t-1}+A^{-t+2}\right)\left(A^{t-T}+A^{T-t+1}\right) \\
= & \left(A^{t}+A^{-t+1}\right)\left(-A^{t-T-1}-A^{T-t+2}\right)+\left(A^{t-1}+A^{-t+2}\right)\left(A^{t-T}+A^{T-t+1}\right) \\
= & -\left(A^{2}-1\right)\left(A^{T}-A^{-T}\right) \\
= & -\rho, \quad t \in[1, T]_{\mathrm{Z}} .
\end{aligned}
$$

Thus, $I_{3} \equiv-1$.

It is easy to check that the corresponding homogeneous problem has only the trivial zero solution. So problem (2.4) has a unique solution $u$ which is given by (2.5). Finally, since $A>1$, we have, for all $(t, s) \in[0, T+1]_{\mathbf{Z}} \times[1, T]_{\mathbf{Z}}, G_{2}(t, s)>0$. The proof is complete.

\section{Estimates on the maximum number of solutions}

In this section, we make estimations on the maximum number of solutions of problem (1.1). First, we prove some lemmas for later use. The first lemma is the discrete Sobolev inequality. 
Lemma 3.1 Assume $u:[0, T+1]_{\mathbf{Z}} \rightarrow \mathbf{R}$ satisfies $\Delta u(T)=0$ and there exists $t_{0} \in[2, T-1]$ such that $u\left(t_{0}\right)=0$. Then

$$
\begin{aligned}
& \sum_{t=t_{0}+1}^{T}|u(t)|^{q} \leq\left(\sum_{t=t_{0}+1}^{T}\left(t-t_{0}\right)^{\frac{q}{p}}\right)\left(\sum_{t=t_{0}+1}^{T}|\Delta u(t-1)|^{q}\right), \\
& \sum_{t=1}^{t_{0}}|u(t)|^{q} \leq\left(\sum_{t=1}^{t_{0}}\left(t_{0}-t\right)^{\frac{q}{p}}\right)\left(\sum_{t=1}^{t_{0}}|\Delta u(t-1)|^{q}\right),
\end{aligned}
$$

where $p>1, \frac{1}{p}+\frac{1}{q}=1$.

Proof For $t>t_{0}$, since $u(t)=\sum_{\tau=t_{0}}^{t-1} \Delta u(\tau)+u\left(t_{0}\right)=\sum_{\tau=t_{0}}^{t-1} \Delta u(\tau)$, we have by the Hölder inequality

$$
|u(t)| \leq \sum_{\tau=t_{0}}^{t-1}|\Delta u(\tau)| \leq\left(t-t_{0}\right)^{\frac{1}{p}}\left(\sum_{\tau=t_{0}}^{t-1}|\Delta u(\tau)|^{q}\right)^{\frac{1}{q}}
$$

which implies that

$$
\sum_{t=t_{0}+1}^{T}|u(t)|^{q} \leq\left(\sum_{t=t_{0}+1}^{T}\left(t-t_{0}\right)^{\frac{q}{p}}\right)\left(\sum_{\tau=t_{0}+1}^{T}|\Delta u(\tau-1)|^{q}\right)
$$

by the boundary condition $\Delta u(T)=0$. On the other hand, for $t<t_{0}$, since $u(t)=u\left(t_{0}\right)-$ $\sum_{\tau=t}^{t_{0}-1} \Delta u(\tau)=-\sum_{\tau=t}^{t_{0}-1} \Delta u(\tau)$, we have

$$
|u(t)| \leq \sum_{\tau=t}^{t_{0}-1}|\Delta u(\tau)| \leq\left(t_{0}-t\right)^{\frac{1}{p}}\left(\sum_{\tau=t}^{t_{0}-1}|\Delta u(\tau)|^{q}\right)^{\frac{1}{q}}
$$

which implies that

$$
\sum_{t=1}^{t_{0}}|u(t)|^{q} \leq\left(\sum_{t=1}^{t_{0}}\left(t_{0}-t\right)^{\frac{q}{p}}\right)\left(\sum_{t=1}^{t_{0}}|\Delta u(t-1)|^{q}\right) .
$$

The proof is complete.

Definition 3.1 We say that $u:[0, T+1]_{\mathbf{Z}} \rightarrow \mathbf{R}$ has a generalized zero at $t_{0} \in[0, T+1]_{\mathbf{Z}}$ provided that $u\left(t_{0}\right)=0$ if $t_{0}=0$ and if $t_{0} \in[1, T+1]_{\mathrm{Z}}$ either $u\left(t_{0}\right)=0$ or $u\left(t_{0}-1\right) u\left(t_{0}\right)<0$.

Lemma 3.2 Let $p:[1, T]_{\mathrm{Z}} \rightarrow \mathbf{R}$ and $p(t) \not \equiv 0$ on $[1, T]_{\mathrm{Z}}$. Consider the following problem:

$$
\left\{\begin{array}{l}
\Delta^{2} u(t-1)+p(t) u(t)=0, \quad t \in[1, T]_{\mathrm{Z}} \\
\Delta u(0)=\Delta u(T)=0
\end{array}\right.
$$

If $p(t)<\frac{4}{T^{2}-1}$ for $t \in[1, T]_{\mathrm{Z}}$, then every nontrivial solution $u(t)$ of (3.1) has no generalized zero on $[0, T+1]_{\mathbf{Z}}$. 
Proof Let $u$ be a nontrivial solution $u(t)$ of (3.1). By the boundary conditions $\Delta u(0)=$ $\Delta u(T)=0$, we know that $u(0)=u(1) \neq 0$ and $u(T)=u(T+1) \neq 0$. Otherwise, $u \equiv 0$ on $[0, T+1]_{\mathrm{Z}}$. Suppose that there exists $t_{0} \in[2, T-1]_{\mathrm{Z}}$ such that $u\left(t_{0}\right)=0$. Multiplying the equation in (3.1) with $u(t)$ and summing both sides from 1 to $t_{0}$, we have

$$
\sum_{t=1}^{t_{0}}[\Delta u(t-1)]^{2}=\sum_{t=1}^{t_{0}} p(t) u^{2}(t) \leq \max _{1 \leq t \leq t_{0}} p_{+}(t) \sum_{t=1}^{t_{0}} u^{2}(t)
$$

where $p_{+}$is the positive part of $p$. Similarly, summing both sides from $t_{0}+1$ to $T$, we have

$$
\sum_{t=t_{0}+1}^{T}[\Delta u(t-1)]^{2}=\sum_{t=t_{0}+1}^{T} p(t) u^{2}(t) \leq \max _{t_{0}+1 \leq t \leq T} p_{+}(t) \sum_{t=t_{0}+1}^{T} u^{2}(t) .
$$

Thus, by Lemma 3.1, we have

$$
\max _{1 \leq t \leq t_{0}} p_{+}(t)\left(\sum_{t=1}^{t_{0}}\left(t_{0}-t\right)\right) \geq 1, \quad \max _{t_{0}+1 \leq t \leq T} p_{+}(t)\left(\sum_{t=t_{0}+1}^{T}\left(t-t_{0}\right)\right) \geq 1 .
$$

It follows that

$$
\max _{1 \leq t \leq T} p_{+}(t) \geq \max \left\{\left(\sum_{t=1}^{t_{0}}\left(t_{0}-t\right)\right)^{-1},\left(\sum_{t=t_{0}+1}^{T}\left(t-t_{0}\right)\right)^{-1}\right\} \geq \frac{4}{T^{2}-1},
$$

which is a contradiction with the assumption $p(t)<\frac{4}{T^{2}-1}$ for $t \in[1, T]_{\mathrm{Z}}$.

Note that the equation $\Delta^{2} u(t-1)+p(t) u(t)=0$ is disconjugate on $\in[0, T+1]_{\mathrm{Z}}$ since $p(t)<\frac{4}{T^{2}-1}$ for $t \in[1, T]_{\mathbf{Z}}$ (see Corollary 6.9 in [30]). It implies that the nontrivial $u$ has at most one generalized zero on $\in[0, T+1]_{\mathbf{Z}}$. Set $z(t)=\frac{u(t+1)}{u(t)}$. Then $z(t)$ satisfies

$$
z(t)+\frac{1}{z(t-1)}=2-p(t), \quad z(0)=z(T)=1
$$

If there exists $t_{0} \in[1, T]_{\mathrm{Z}}$ such that $z\left(t_{0}\right)<0$, then the boundary conditions $z(0)=z(T)=1$ implies that $z(t)$ has at least two generalized zeros. Thus, $u(t)$ has at least two generalized zeros, which contradicts with that $u(t)$ is disconjugate. The proof is complete.

Lemma 3.3 Let $p_{1}, p_{2}:[1, T]_{\mathbf{Z}} \rightarrow \mathbf{R}$ satisfy the conditions of Lemma 3.2. Assume $p_{1}(t) \leq$ $p_{2}(t)$ on $[1, T]_{\mathrm{Z}}$ and there exists $t_{0} \in[1, T]_{\mathrm{Z}}$ such that $p_{1}\left(t_{0}\right)<p_{2}\left(t_{0}\right)$. Then at least one of the problems

$$
\left\{\begin{array}{l}
\Delta^{2} u(t-1)+p_{i}(t) u(t)=0, \quad t \in[1, T]_{\mathrm{Z}}, \quad i=1,2, \\
\Delta u(0)=\Delta u(T)=0,
\end{array}\right.
$$

has only the trivial solution.

Proof By way of contradiction, assume that $u_{1}$ and $u_{2}$ are the nontrivial solutions of problems (3.2) corresponding to $p_{1}$ and $p_{2}$, respectively. By Lemma 3.2, we know that $u_{1}(t)$ and 
$u_{2}(t)$ both have no generalized zero on $t \in[0, T+1]_{\mathbf{Z}}$. It follows by $p_{1}(t)-p_{2}(t) \leq 0$ and $p_{1}(t)-p_{2}(t) \not \equiv 0, t \in[1, T]_{\mathrm{Z}}$, that

$$
\sum_{t=1}^{T}\left[p_{1}(t)-p_{2}(t)\right] u_{1}(t) u_{2}(t) \neq 0 .
$$

On the other hand, by

$$
\begin{aligned}
& u_{2}(t)\left[\Delta^{2} u_{1}(t-1)+p_{1}(t) u_{1}(t)\right]=0, \\
& u_{1}(t)\left[\Delta^{2} u_{2}(t-1)+p_{2}(t) u_{2}(t)\right]=0,
\end{aligned}
$$

we have

$$
\left[u_{2}(t) \Delta^{2} u_{1}(t-1)-u_{1}(t) \Delta^{2} u_{2}(t-1)\right]+\left[p_{1}(t)-p_{2}(t)\right] u_{1}(t) u_{2}(t)=0 .
$$

Summing both sides from 1 to $T$, we get

$$
\sum_{t=1}^{T}\left[p_{1}(t)-p_{2}(t)\right] u_{1}(t) u_{2}(t)=0,
$$

which is a contradiction with (3.3). The proof is complete.

Lemma 3.4 Assume $g \in C^{1}(\mathbf{R})$ and $g^{\prime}(x)<\frac{4}{T^{2}-1}$ for all $x \in \mathbf{R}$. Let $u_{1}$ and $u_{2}$ are two distinct solutions of $(1.1)$, then $u_{1}(t)-u_{2}(t)$ has no generalized zero on $[0, T+1]_{Z}$ and hence $u_{1}$ and $u_{2}$ are strictly ordered.

Proof Set $v=u_{1}-u_{2}$. Then $v$ is a nontrivial solution of (3.1) with

$$
p(t)= \begin{cases}\frac{g\left(u_{1}(t)\right)-g\left(u_{2}(t)\right)}{u_{1}(t)-u_{2}(t)}, & u_{1}(t) \neq u_{2}(t), \\ g^{\prime}\left(u_{1}(t)\right), & u_{1}(t)=u_{2}(t) .\end{cases}
$$

Since $g^{\prime}(x)<\frac{4}{T^{2}-1}$, we know $p(t)<\frac{4}{T^{2}-1}$ for all $t \in[1, T]_{\mathrm{Z}}$. Thus, Lemma 3.2 implies that $v(t)$ has no generalized zero on $[0, T+1]_{\mathrm{Z}}$. Therefore, $u_{1}$ and $u_{2}$ are strictly ordered. The proof is complete.

Now, we show our main results of this section. First, if $g$ is strictly convex on $\mathbf{R}$, we have the following result.

Theorem 3.5 Assume $g \in C^{1}(\mathbf{R}), g^{\prime}(x)<\frac{4}{T^{2}-1}$ for all $x \in \mathbf{R}$ and $g^{\prime}$ is strictly increasing. Then problem (1.1) has at most two solutions.

Proof Suppose that problem (1.1) has three solutions $u_{1}, u_{2}$, and $u_{3}$. By Lemma 3.4, we assume $u_{1}(t)<u_{2}(t)<u_{3}(t), t \in[0, T+1]_{\mathrm{Z}}$. Let $v_{1}=u_{2}-u_{1}, v_{2}=u_{3}-u_{2}$, then $v_{1}$ and $v_{2}$ solve problem (3.1) with, respectively,

$$
p(t)=p_{1}(t):=\frac{g\left(u_{2}(t)\right)-g\left(u_{1}(t)\right)}{u_{2}(t)-u_{1}(t)}
$$


and

$$
p(t)=p_{2}(t):=\frac{g\left(u_{3}(t)\right)-g\left(u_{2}(t)\right)}{u_{3}(t)-u_{2}(t)} .
$$

Since $g^{\prime}$ is strictly increasing, we have $p_{1}(t)<p_{2}(t)<\frac{4}{T^{2}-1}$. By Lemma 3.3, either $v_{1}(t) \equiv 0$ or $v_{2}(t) \equiv 0$, which is a contradiction. The proof is complete.

Similarly, if $g$ is strictly concave on $\mathbf{R}$, we have the following result.

Theorem 3.6 Assume $g \in C^{1}(\mathbf{R}), g^{\prime}(x)<\frac{4}{T^{2}-1}$ for all $x \in \mathbf{R}$ and $g^{\prime}$ is strictly decreasing. Then problem (1.1) has at most two solutions.

\section{On the minimum number of solutions and positive solutions}

In this section, we consider the minimum numbers of solution and positive solutions of (1.1). First, we consider the nonexistence, existence and multiplicity of solutions of (1.1) when $g \in C^{1}(\mathbf{R})$ and $g$ has a unique global minimum value on $\mathbf{R}$.

Theorem 4.1 Let $g \in C^{1}(\mathbf{R}), \lim _{x \rightarrow-\infty} g(x)=a, \lim _{x \rightarrow+\infty} g(x)=b$, and $\lambda_{*}:=g\left(x_{0}\right)=$ $\min _{x \in \mathbf{R}} g(x)$. Here, $a, b$ may be $+\infty$. Assume $g(x)>\lambda_{*}$ for all $x \in \mathbf{R} \backslash\left\{x_{0}\right\}$ and $\lambda_{*}<\min \{a, b\}$.

(1) If one of the following conditions is satisfied, then problem (1.1) has no solution:

(1-i) $h(t) \leq \lambda_{*}$ and $h(t) \not \equiv \lambda_{*}$ for $t \in[1, T]_{\mathrm{Z}}$;

(1-ii) $\max \{a, b\}<+\infty, h(t) \geq \max \{a, b\}$ for all $t \in[1, T]_{\mathrm{Z}}$ and $\max \{a, b\}>g(x)$ for all $x \in \mathbf{R}$.

(2) If $h(t) \equiv \lambda_{*}$ on $[1, T]_{\mathbf{Z}}$, then problem (1.1) has exactly one solution.

(3) If $\lambda_{*} \leq h(t)<\min \{a, b\}, h(t) \neq \lambda_{*}$ for $t \in[1, T]_{\mathrm{Z}}$, and $g^{\prime}(x)<4 \sin ^{2} \frac{\pi}{4 T}$ on $\left[x_{0}, \infty\right)$, then problem (1.1) has at least two solutions.

(4) If one of the following conditions is satisfied, then problem (1.1) has at least one solution:

(4-i) $a \neq b, \min \{a, b\}<+\infty$ and $h(t) \equiv \min \{a, b\}, t \in[1, T]_{\mathbf{Z}}$;

(4-ii) $b<h(t)<a, t \in[1, T]_{\mathbf{Z}}$

(4-iii) $a<h(t)<b, t \in[1, T]_{\mathrm{Z}}$, and $g^{\prime}(x)<4 \sin ^{2} \frac{\pi}{4 T}$ on $\left[x_{0}, \infty\right)$.

Proof (1-i) Since $h(t) \leq \lambda_{*}$ and $h(t) \not \equiv \lambda_{*}$ for $t \in[1, T]_{\mathrm{Z}}$, we have $\sum_{t=1}^{T} h(t)<T \lambda_{*}$. Assume problem (1.1) has a solution $u$. Since $g(u(t)) \geq \lambda_{*}, t \in[1, T]_{\mathrm{Z}}, \sum_{t=1}^{T} g(u(t)) \geq T \lambda_{*}$. Summing both sides of the equation $\Delta^{2} u(t-1)+g(u(t))=h(t)$ from 1 to $T$, we have by the boundary conditions $\Delta u(0)=\Delta u(T)=0$,

$$
T \lambda_{*} \leq \sum_{t=1}^{T} g(u(t))=\sum_{t=1}^{T} h(t)<T \lambda_{*},
$$

which is a contradiction.

(1-ii) Suppose (1.1) has a solution $u$. Then $g(u(t))<\max \{a, b\}$ for all $t \in[1, T]_{\mathrm{Z}}$, which implies that $\Delta^{2} u(t-1)=h(t)-g(u(t))>0$ for $t \in[1, T]_{\mathrm{Z}}$. Thus, we have the contradiction that $\Delta u(T)>\Delta u(0)$.

(2) It is easy to see that $u(t) \equiv x_{0}$ is a solution of (1.1). Assume that $v$ is also a solution of (1.1). Since $g(v(t)) \geq \lambda_{*}, t \in[1, T]_{\mathrm{Z}}$, we have $\Delta^{2} v(t-1) \leq 0, t \in[1, T]_{\mathrm{Z}}$. It follows by the 
boundary conditions $\Delta v(0)=\Delta v(T)=0$ that $\Delta v(t) \equiv 0, t \in[0, T]_{\mathrm{Z}}$. Thus, $g(v(t)) \equiv g\left(x_{0}\right)$. Therefore, $v(t) \equiv x_{0}, t \in[0, T+1]_{\mathbf{Z}}$, since $g(x)>g\left(x_{0}\right)$ for all $x \in \mathbf{R} \backslash\left\{x_{0}\right\}$.

(3) Without loss of generality, we assume $b \leq a$. Since $\lambda_{*} \leq h(t)<b, h(t) \not \equiv \lambda_{*}, t \in[1, T]_{\mathrm{Z}}$, we see by $\lim _{x \rightarrow-\infty} g(x)=a$ and $\lim _{x \rightarrow+\infty} g(x)=b$ that there exist $c_{1}<x_{0}<c_{2}$ such that

$$
g\left(c_{1}\right)>h(t), \quad g\left(c_{2}\right)>h(t), \quad t \in[1, T]_{\mathbf{Z}}
$$

Set $u_{1}(t) \equiv c_{1}, u_{2}(t) \equiv x_{0}, u_{3}(t) \equiv c_{2}, t \in[0, T+1]_{\mathrm{Z}}$. We prove that problem (1.1) has at least two solutions $u_{1}^{*}, u_{2}^{*}: u_{1}^{*} \in\left[u_{1}, u_{2}\right], u_{2}^{*} \in\left[u_{2}, u_{3}\right]$. Here, $\left[u_{1}, u_{2}\right]$ and $\left[u_{2}, u_{3}\right]$ denote order intervals.

First, we show that $u_{2}(t)$ is not a solution of (1.1). In fact, if it is not true, then summing both sides of the equation of (1.1) yields a contradiction:

$$
T \lambda_{*}=\sum_{t=1}^{T} g\left(u_{2}(t)\right)=\sum_{t=1}^{T} h(t)>T \lambda_{*} .
$$

Second, we prove that (1.1) has at least one solution $u_{1}^{*} \in\left[u_{1}, u_{2}\right]$. Let $E=\{u:[0, T+$ $\left.1]_{\mathbf{Z}} \rightarrow \mathbf{R}\right\}$ with the norm $\|u\|=\max _{t \in[0, T+1]_{\mathbf{Z}}}|u(t)|$. Since $g^{\prime}$ is continuous on the bounded closed interval $\left[c_{1}, x_{0}\right]$, there exists $L>0$ such that $g^{\prime}(x)>-L$ for $x \in\left[c_{1}, x_{0}\right]$. Consider the following problem:

$$
\left\{\begin{array}{l}
-\Delta^{2} u(t-1)+L u(t)=g(u(t))+L u(t)-h(t), \quad t \in[1, T]_{\mathrm{Z}} \\
\Delta u(0)=\Delta u(T)=0 .
\end{array}\right.
$$

By Lemma 2.2, (4.2) is equivalent to $u(t)=S u(t)$. Here, $S: E \rightarrow E$ is defined by

$$
S u(t)=\sum_{s=1}^{T} G_{2}(t, s)[g(u(s))+L u(s)-h(s)], \quad t \in[0, T+1]_{\mathbf{Z}} .
$$

It is easy to see that $S$ is continuous. Since $u_{1}(t)$ satisfies

$$
\left\{\begin{array}{l}
-\Delta^{2} u(t-1)+L u(t)=L u_{1}(t), \quad t \in[1, T]_{\mathrm{Z}}, \\
\Delta u(0)=\Delta u(T)=0,
\end{array}\right.
$$

we see by (4.1) and the positivity of $G_{2}(t, s)$ that

$$
\begin{aligned}
S u_{1}(t) & =\sum_{s=1}^{T} G_{2}(t, s)\left[g\left(u_{1}(s)\right)+L u_{1}(s)-h(s)\right] \\
& >\sum_{s=1}^{T} G_{2}(t, s) L u_{1}(s)=u_{1}(t), \quad t \in[0, T+1]_{\mathrm{Z}}
\end{aligned}
$$

which shows that $u_{1}$ is a lower solution of the operator $S$. Similarly, one can check that $S u_{2}(t) \leq u_{2}(t)$ and hence $u_{2}$ is an upper solution of $S$. On the other hand, $S$ is an increasing operator defined on $\left[u_{1}, u_{2}\right]$. In fact, for $v_{1}, v_{2} \in\left[u_{1}, u_{2}\right]$ with $v_{1} \leq v_{2}$, we have

$$
\begin{aligned}
S v_{2}(t)-S v_{1}(t) & =\sum_{s=1}^{T} G_{2}(t, s)\left[g\left(v_{2}(s)\right)-g\left(v_{1}(s)\right)+L\left(v_{2}(s)-v_{1}(s)\right)\right] \\
& \geq 0, \quad t \in[0, T+1]_{\mathbf{Z}}
\end{aligned}
$$


by $g^{\prime}(x)>-L$ for $x \in\left[c_{1}, x_{0}\right]$. Therefore, $S$ has at least one solution $u_{1}^{*} \in\left[u_{1}, u_{2}\right]$ by the fixed point theorem of increasing operator in ordered Banach spaces due to Amann [31]. So, (4.2), and hence (1.1), has at least one solution $u_{1}^{*} \in\left[u_{1}, u_{2}\right]$.

Now, we prove that (1.1) has at least one solution $u_{2}^{*} \in\left[u_{2}, u_{3}\right]$. Since $g^{\prime}(x)<4 \sin ^{2} \frac{\pi}{4 T}$ for $x \in\left[x_{0},+\infty\right)$, there exists $K>0$ such that

$$
g^{\prime}(x)<K<4 \sin ^{2} \frac{\pi}{4 T}, \quad x \in\left[x_{0}, c_{2}\right]
$$

Consider the following problem:

$$
\left\{\begin{array}{l}
\Delta^{2} u(t-1)+K u(t)=h(t)-g(u(t))+K u(t), \quad t \in[1, T]_{\mathbf{Z}} \\
\Delta u(0)=\Delta u(T)=0 .
\end{array}\right.
$$

By Lemma 2.1, $G_{1}(t, s)>0$, and (4.5) is equivalent to $u(t)=Q u(t)$. Here, $Q: E \rightarrow E$ is defined by

$$
Q u(t)=\sum_{s=1}^{T} G_{1}(t, s)[h(s)-g(u(s))+K u(s)], \quad t \in[0, T+1]_{\mathbf{Z}} .
$$

Similar to the discussion of the operator $S, Q$ has at least one solution $u_{2}^{*} \in\left[u_{2}, u_{3}\right]$, which is a solution of (1.1). Therefore, problem (1.1) has at least two solutions.

(4-i) Without loss of generality, we assume $b<a$. Since $h(t) \equiv b, t \in[1, T]_{\mathrm{Z}}$, there exists $c_{1}<x_{0}$ such that $g\left(c_{1}\right)=b$. Obviously, $u(t) \equiv c_{1}$ is a solution of (1.1).

(4-ii) Since $b<h(t)<a, t \in[1, T]_{\mathrm{Z}}$, there exist $c_{1}<c_{2}<x_{0}$ such that $g\left(c_{1}\right)>h(t), g\left(c_{2}\right)<$ $h(t), t \in[1, T]_{\mathrm{Z}}$. By the continuity of $g^{\prime}$, there exists $L>0$ such that $g^{\prime}(x)>-L, x \in\left[c_{1}, c_{2}\right]$. Let $u_{1}(t) \equiv c_{1}, u_{2}(t) \equiv c_{2}$. Considering the operator $S$ defined as (4.3) on $\left[u_{1}, u_{2}\right]$, one can see that $S$ has a fixed point $u^{*} \in\left[u_{1}, u_{2}\right]$, which is a solution of (1.1).

(4-iii) Since $a<h(t)<b, t \in[1, T]_{\mathrm{Z}}$, there exist $x_{0}<c_{1}<c_{2}$ such that $g\left(c_{1}\right)<h(t), g\left(c_{2}\right)>$ $h(t), t \in[1, T]_{\mathrm{Z}}$. By $g^{\prime}(x)<4 \sin ^{2} \frac{\pi}{4 T}$ for $x \geq x_{0}$, there exists $K>0$ such that $g^{\prime}(x)<K<$ $4 \sin ^{2} \frac{\pi}{4 T}, x \in\left[c_{1}, c_{2}\right]$. Let $u_{1}(t) \equiv c_{1}, u_{2}(t) \equiv c_{2}$. Considering the operator $Q$ defined as (4.5) on $\left[u_{1}, u_{2}\right]$, one can see that $Q$ has a fixed point $u^{*} \in\left[u_{1}, u_{2}\right]$, which is a solution of (1.1).

The proof is complete.

Similarly, if $g \in C^{1}(\mathbf{R})$ and $g$ has a unique global maximum value on $\mathbf{R}$, we have the following result.

Theorem 4.2 Let $g \in C^{1}(\mathbf{R}), \lim _{x \rightarrow-\infty} g(x)=a, \lim _{x \rightarrow+\infty} g(x)=b$, and $\lambda^{*}:=g\left(x_{0}\right)=$ $\max _{x \in \mathbf{R}} g(x)$. Here, $a, b$ may be $-\infty$. Assume $g(x)<\lambda^{*}$ for all $x \in \mathbf{R} \backslash\left\{x_{0}\right\}$ and $\lambda^{*}>\max \{a, b\}$.

(1) If one of the following conditions is satisfied, then problem (1.1) has no solution:

(1-i) $h(t) \geq \lambda^{*}$ and $h(t) \not \equiv \lambda^{*}$ for $t \in[1, T]_{\mathrm{Z}}$;

(1-ii) $\min \{a, b\}>-\infty, h(t) \leq \min \{a, b\}$ for $t \in[1, T]_{\mathrm{Z}}$ and $\min \{a, b\}<g(x)$ for $x \in \mathbf{R}$.

(2) If $h(t) \equiv \lambda^{*}$ on $[1, T]_{\mathbf{Z}}$, then problem (1.1) has exactly one solution.

(3) If $\max \{a, b\}<h(t) \leq \lambda^{*}, h(t) \not \equiv \lambda^{*}$ for $t \in[1, T]_{\mathrm{Z}}$; and $g^{\prime}(x)<4 \sin ^{2} \frac{\pi}{4 T}$ on $\left(-\infty, x_{0}\right]$, then problem (1.1) has at least two solutions.

(4) If one of the following conditions is satisfied, then problem (1.1) has at least one solution: 
(4-i) $a \neq b, \max \{a, b\}>-\infty$ and $h(t) \equiv \max \{a, b\}$ for $t \in[1, T]_{\mathrm{z}}$;

(4-ii) $a<h(t)<b$ for $t \in[1, T]_{\mathrm{Z}}$, and $g^{\prime}(x)<4 \sin ^{2} \frac{\pi}{4 T}$ on $\left(-\infty, x_{0}\right]$;

(4-iii) $b<h(t)<a$ for $t \in[1, T]_{\mathrm{Z}}$.

Now, we consider the positive solutions of $(1.1)$. Let $\langle 0,+\infty)$ denote $(0,+\infty)$ or $[0, \infty)$. First, if $g \in C^{1}(\langle 0,+\infty), \mathbf{R})$ and $g$ has a unique global minimum value on $\langle 0,+\infty)$, we have the following result.

Theorem 4.3 Let $g \in C^{1}(\langle 0,+\infty), \mathbf{R}), \lim _{x \rightarrow 0^{+}} g(x)=a, \lim _{x \rightarrow+\infty} g(x)=b$. Here, $a, b$ may be $+\infty$. Assume that there exists $x_{0}>0$ such that

$$
\lambda_{*}:=g\left(x_{0}\right)=\min _{x \in\langle 0,+\infty)} g(x),
$$

and $g(x)>\lambda_{*}$ for all $x \in\langle 0,+\infty) \backslash\left\{x_{0}\right\}, \lambda_{*}<\min \{a, b\}$.

(I) Suppose $\lambda_{*} \geq 0$.

(1) If one of the following conditions is satisfied, then problem (1.1) has no positive solution:

$(1-\mathrm{i}) \quad h(t) \leq \lambda_{*}$ and $h(t) \not \equiv \lambda_{*}$ for $t \in[1, T]_{\mathrm{Z}}$;

(1-ii) $\max \{a, b\}<+\infty, h(t) \geq \max \{a, b\}$ for $t \in[1, T]_{\mathrm{Z}}$, and $g(x)<\max \{a, b\}$ for all $x \in(0,+\infty)$.

(2) If $h(t) \equiv \lambda_{*}$ for $t \in[1, T]_{\mathbf{Z}}$, then problem (1.1) has exactly one positive solution.

(3) If $\lambda_{*} \leq h(t)<\min \{a, b\}, h(t) \not \equiv \lambda_{*}$ for $t \in[1, T]_{\mathrm{Z}}$, and $g^{\prime}(x)<4 \sin ^{2} \frac{\pi}{4 T}$ on $\left[x_{0},+\infty\right)$, then problem (1.1) has at least two positive solutions.

(4) If one of the following conditions is satisfied, then problem (1.1) has at least one positive solution:

(4-i) $a \neq b, \min \{a, b\}<+\infty, h(t) \equiv \min \{a, b\}$ for $t \in[1, T]_{\mathrm{Z}}$

(4-ii) $b<h(t)<a$ for $t \in[1, T]_{\mathrm{Z}}$;

(4-iii) $a<h(t)<$ for $t \in[1, T]_{\mathrm{Z}}$, and $g^{\prime}(x)<4 \sin ^{2} \frac{\pi}{4 T}$ on $\left[x_{0}, \infty\right)$.

(II) Suppose $\lambda_{*}<0$.

(5) If one of the following conditions is satisfied, then problem (1.1) has no positive solution:

(5-i) $h(t) \leq \lambda_{*}$ for $t \in[1, T]_{\mathbf{Z}}$;

(5-ii) $\max \{a, b\}<+\infty$ and $h(t) \geq \max \{a, b\}$ for $t \in[1, T]_{\mathrm{Z}}$, and $g(x)<\max \{a, b\}$ for all $x \in(0,+\infty)$.

(6) If one of the following conditions is satisfied, then problem (1.1) has at least two positive solutions:

(6-i) $a>0, b>0,0<h(t)<\min \{a, b\}$ for $t \in[1, T]_{\mathrm{Z}}$, and $g^{\prime}(x)<4 \sin ^{2} \frac{\pi}{4 T}$ on $\left[x_{0}, \infty\right)$;

(6-ii) $a>0, b>0$, and $h(t) \equiv 0$ for $t \in[1, T]_{\mathrm{z}}$.

(7) If one of the following conditions is satisfied, then problem (1.1) has at least one positive solution:

(7-i) $0<\min \{a, b\}<+\infty, a \neq b$, and $h(t) \equiv \min \{a, b\}$ for $t \in[1, T]_{\mathrm{Z}}$;

(7-ii) $0<b<a$ and $b<h(t)<a$ for $t \in[1, T]_{\mathrm{Z}}$;

(7-iii) $0<a<b, a<h(t)<b$ for $t \in[1, T]_{\mathrm{Z}}$, and $g^{\prime}(x)<4 \sin ^{2} \frac{\pi}{4 T}$ on $\left[x_{0}, \infty\right)$;

(7-iv) $a \leq 0, b>0$, and $h(t) \equiv 0$ for $t \in[1, T]_{\mathrm{Z}}$;

(7-v) $a \leq 0, b>0,0<h(t)<b$ for $t \in[1, T]_{\mathrm{Z}}$, and $g^{\prime}(x)<4 \sin ^{2} \frac{\pi}{4 T}$ on $\left[x_{0}, \infty\right)$;

(7-vi) $a>0, b<0$, and $0<h(t)<a$ for $t \in[1, T]_{\mathrm{Z}}$. 
Proof We only show the proofs of (3), (6), and (7-iv)-(7-vi).

(3) Without loss of generality, we assume $b \leq a$. By the proof of Theorem 4.1(3), we can choose $0<c_{1}<x_{0}<c_{2}$ and $L>0, K>0$, such that $S$ defined as (4.3) has a fixed point $u_{1}^{*}$ satisfying $c_{1} \leq u_{1}^{*}(t) \leq x_{0}$ and $Q$ defined as (4.5) has a fixed point $u_{2}^{*}$ satisfying $x_{0} \leq u_{2}^{*}(t) \leq$ $c_{2}$. Clearly, $u_{1}^{*}(t)$ and $u_{2}^{*}(t)$ are two positive solutions of (1.1).

(6) By $a>0, b>0$, and $\lambda_{*}<0$, there exist $x_{1}$ and $x_{2}$ satisfying $0<x_{1}<x_{0}<x_{2}$ such that $g\left(x_{1}\right)=g\left(x_{2}\right)=0$. Therefore, if $h(t) \equiv 0$ on $[1, T]_{\mathrm{Z}}$, then $u(t) \equiv x_{1}$ and $u(t) \equiv x_{2}$ are two positive solutions of (1.1). If $0<h(t)<\min \{a, b\}$, we know that there exist $0<c_{1}<c_{2}<x_{1}$ and $x_{2}<c_{3}<c_{4}$ such that

$$
g\left(c_{1}\right)>h(t), \quad g\left(c_{2}\right)<h(t), \quad g\left(c_{3}\right)<h(t), \quad g\left(c_{4}\right)>h(t) .
$$

Let $u_{i}(t) \equiv c_{i}(i=1,2,3,4)$. Similar to the proof of Theorem 4.1(3), consider the operator $S$ defined as (4.3) on $\left[u_{1}, u_{2}\right]$ and the operator $Q$ defined as (4.5) on $\left[u_{3}, u_{4}\right]$, one can find that $S$ has a fixed point in $\left[u_{1}, u_{2}\right]$ and $Q$ has a fixed point in $\left[u_{3}, u_{4}\right]$.

(7-iv) By $a \leq 0, b>0$, then there exists $x_{1}>x_{0}$ by $\lambda_{*}<0$ such that $g\left(x_{1}\right)=0$. It is easy to see that $u(t) \equiv x_{1}$ is a positive solution of (1.1) since $h(t) \equiv 0$ for $t \in[1, T]_{\mathbf{Z}}$.

(7-v) By $a \leq 0, b>0$, there exists $x_{1}>0$ such that $g\left(x_{1}\right)=0$. Thus, $0<h(t)<b, t \in[1, T]_{\mathrm{Z}}$, implies that there exist $c_{2}>c_{1}>x_{1}$ such that $g\left(c_{1}\right)<h(t)$ and $g\left(c_{2}\right)>h(t)$ for $t \in[1, T]_{\mathbf{z}}$. Similar to the proof of Theorem 4.1(3), $Q$ defined on $\left[u_{1}, u_{2}\right]$ has a fixed point in $\left[u_{1}, u_{2}\right]$, where $u_{1}(t) \equiv c_{1}, u_{2}(t) \equiv c_{2}, t \in[1, T]_{\mathbf{Z}}$.

(7-vi) By $a>0, b<0$, there exists $x_{1}>0$ such that $g\left(x_{1}\right)=0$. Since $0<h(t)<a$ for $t \in$ $[1, T]_{\mathrm{Z}}$, there exist $0<c_{1}<c_{2}<x_{1}$ such that $g\left(c_{1}\right)>h(t)$ and $g\left(c_{2}\right)<h(t)$ for $t \in[1, T]_{\mathrm{Z}}$. Similar to the proof of Theorem 4.1(3), $S$ defined on $\left[u_{1}, u_{2}\right]$ has a fixed point in $\left[u_{1}, u_{2}\right]$, where $u_{1}(t) \equiv c_{1}, u_{2}(t) \equiv c_{2}, t \in[1, T]_{\mathbf{Z}}$.

The proof is complete.

Finally, if $g \in C^{1}(\langle 0,+\infty), \mathbf{R})$ and $g$ has a unique global maximum value on $\langle 0,+\infty)$, we have the following result.

Theorem 4.4 Let $g \in C^{1}(\langle 0,+\infty), \mathbf{R}), \lim _{x \rightarrow 0^{+}} g(x)=a$, $\lim _{x \rightarrow+\infty} g(x)=b$. Here, $a, b$ may $b e-\infty$. Assume that there exists $x_{0}>0$ such that

$$
\lambda^{*}:=g\left(x_{0}\right)=\max _{x \in\langle 0,+\infty)} g(x)
$$

and $g(x)<\lambda^{*}$ for all $x \in\langle 0,+\infty) \backslash\left\{x_{0}\right\}, \lambda^{*}>0$, and $\lambda^{*}>\min \{a, b\}$.

(1) If one of the following conditions is satisfied, then problem (1.1) has no positive solution:

(1-i) $h(t) \geq \lambda^{*}$ and $h(t) \not \equiv \lambda^{*}$ for $t \in[1, T]_{\mathrm{Z}}$;

(1-ii) $\min \{a, b\} \geq 0, h(t) \leq \min \{a, b\}$ for $t \in[1, T]_{\mathrm{Z}}$, and $g(x)>\min \{a, b\}$ for all $x \in(0,+\infty)$.

(2) If $h(t) \equiv \lambda^{*}$ for $t \in[1, T]_{\mathrm{Z}}$, then problem (1.1) has exactly one positive solution.

(3) If one of the following conditions is satisfied, then problem (1.1) has at least two positive solutions:

(3-i) $\max \{a, b, 0\}<h(t) \leq \lambda^{*}, h(t) \not \equiv \lambda_{*}$ for $t \in[1, T]_{\mathrm{Z}}$, and $g^{\prime}(x)<4 \sin ^{2} \frac{\pi}{4 T}$ on $\left(0, x_{0}\right)$;

(3-ii) $a<0, b<0$, and $h(t) \equiv 0$ for $t \in[1, T]_{\mathbf{Z}}$. 
(4) If one of the following conditions is satisfied, then problem (1.1) has at least one positive solution:

(4-i) $\max \{a, b\}>0, a \neq b$, and $h(t) \equiv \max \{a, b\}$ for $t \in[1, T]_{\mathrm{Z}}$;

(4-ii) $a>0, b<0$, and $0<h(t)<a$ for $t \in[1, T]_{\mathrm{Z}}$;

(4-iii) $a<0, b>0,0<h(t)<b$ for $t \in[1, T]_{\mathrm{z}}$, and $g^{\prime}(x)<4 \sin ^{2} \frac{\pi}{4 T}$ on $\left(0, x_{0}\right)$;

(4-iv) either $a \geq 0, b<0$ or $a<0, b \geq 0$, and $h(t) \equiv 0$ for $t \in[1, T]_{\mathrm{Z}}$.

\section{Exact numbers of solutions and positive solutions}

In this section, we establish the results of exact multiplicities of solutions and positive solutions for problem (1.1). First, we consider the exact number of solutions of (1.1).

Note that $4 \sin ^{2} \frac{\pi}{4 T}<\frac{4}{T^{2}-1}$ for $T>3$.

If $g \in C^{1}(\mathbf{R})$ and $g$ is strictly convex on $\mathbf{R}$, we have the following result by Theorem 3.5 and Theorem 4.1.

Theorem 5.1 Assume $g \in C^{1}(\mathbf{R}), g^{\prime}(x)$ is strictly increasing and $g^{\prime}(x)<4 \sin ^{2} \frac{\pi}{4 T}$ for all $x \in \mathbf{R}$. Let

$$
\lim _{x \rightarrow \pm \infty} g(x)=+\infty, \quad \lambda_{*}=\min _{x \in \mathbf{R}} g(x) .
$$

(1) If $h(t) \leq \lambda_{*}$ and $h(t) \not \equiv \lambda_{*}$ for $t \in[1, T]_{\mathrm{Z}}$, then problem (1.1) has no solution.

(2) If $h(t) \equiv \lambda_{*}$ for $t \in[1, T]_{\mathbf{Z}}$, then problem (1.1) has exactly one solution.

(3) If $h(t) \geq \lambda_{*}$ and $h(t) \not \equiv \lambda_{*}$ for $t \in[1, T]_{\mathrm{z}}$, then problem (1.1) has exactly two solutions.

If $g \in C^{1}(\mathbf{R})$ and $g$ is strictly concave on $\mathbf{R}$, then, by Theorem 3.6 and Theorem 4.2, we have the following result.

Theorem 5.2 Assume $g \in C^{1}(\mathbf{R}), g^{\prime}(x)$ is strictly decreasing, and $g^{\prime}(x)<4 \sin ^{2} \frac{\pi}{4 T}$ for all $x \in \mathbf{R}$. Let

$$
\lim _{x \rightarrow \pm \infty} g(x)=-\infty, \quad \lambda^{*}=\max _{x \in \mathbf{R}} g(x)
$$

(1) If $h(t) \geq \lambda^{*}$ and $h(t) \not \equiv \lambda^{*}$ for $t \in[1, T]_{\mathbf{Z}}$, then problem (1.1) has no solution.

(2) If $h(t) \equiv \lambda^{*}$ for $t \in[1, T]_{\mathbf{Z}}$, then problem (1.1) has exactly one solution.

(3) If $h(t) \leq \lambda^{*}$ and $h(t) \not \equiv \lambda^{*}$ for $t \in[1, T]_{\mathrm{Z}}$, then problem (1.1) has exactly two solutions.

Now, we consider the exact number of positive solutions of problem (1.1). First, we consider the case that $g \in C^{1}(\langle 0,+\infty), \mathbf{R})$ and $g$ is strictly convex on $\langle 0,+\infty)$. If $g \in$ $C^{1}((0, \infty), \mathbf{R})$, we have the following result by Theorem 3.5 and Theorem 4.3 .

Theorem 5.3 Assume $g \in C^{1}((0, \infty), \mathbf{R}), g^{\prime}(x)$ is strictly increasing, and $g^{\prime}(x)<4 \sin ^{2} \frac{\pi}{4 T}$ for all $x \in(0, \infty)$. Let

$$
\lim _{x \rightarrow 0} g(x)=\lim _{x \rightarrow+\infty} g(x)=+\infty, \quad \lambda_{*}=\min _{x \in(0,+\infty)} g(x) .
$$

(1) If $h(t) \leq \lambda_{*}$ and $h(t) \not \equiv \lambda_{*}$ for $t \in[1, T]_{\mathrm{Z}}$, then problem (1.1) has no positive solution.

(2) If $h(t) \equiv \lambda_{*}$ for $t \in[1, T]_{\mathbf{Z}}$, then problem (1.1) has exactly one positive solution. 
(3) If one of the following conditions is satisfied, then problem (1.1) has exactly two positive solutions:

(i) $\lambda_{*} \geq 0, h(t) \geq \lambda_{*}$, and $h(t) \not \equiv \lambda_{*}$ for $t \in[1, T]_{\mathrm{Z}}$;

(ii) $\lambda_{*}<0$ and $h(t) \equiv 0$ for $t \in[1, T]_{\mathrm{Z}}$;

(iii) $\lambda_{*}<0$ and $h(t)>0$ for $t \in[1, T]_{\mathbf{Z}}$.

If $g \in C^{1}([0, \infty), \mathbf{R})$, we have the following result.

Theorem 5.4 Assume $g \in C^{1}([0, \infty), \mathbf{R}), g^{\prime}(x)$ is strictly increasing and $g^{\prime}(x)<4 \sin ^{2} \frac{\pi}{4 T}$ for all $x \in[0, \infty)$. Let

$$
g(0)=a, \quad \lim _{x \rightarrow+\infty} g(x)=+\infty, \quad \lambda_{*}=\min _{x \in[0,+\infty)} g(x) .
$$

(I) Suppose $\lambda_{*} \geq 0$.

(1) If $h(t) \leq \lambda_{*}$ and $h(t) \not \equiv \lambda_{*}$ for $t \in[1, T]_{\mathrm{Z}}$, then problem (1.1) has no positive solution.

(2) If one of the following conditions is satisfied, then problem (1.1) has exactly one positive solution:

(2-i) $\lambda_{*}<a$ and $h(t) \equiv \lambda_{*}$ for $t \in[1, T]_{\mathbf{Z}}$;

(2-ii) $\lambda_{*}<a$ and $h(t) \equiv$ a for $t \in[1, T]_{\mathrm{Z}}$;

(2-iii) $h(t)>$ a for $t \in[1, T]_{\mathbf{Z}}$.

(3) If $\lambda_{*} \leq h(t)<a$ and $h(t) \not \equiv \lambda_{*}$ for $t \in[1, T]_{\mathbf{Z}}$, then problem (1.1) has exactly two positive solutions.

(II) Suppose $\lambda_{*}<0$.

(4) If one of the following conditions is satisfied, then problem (1.1) has exactly one positive solution:

(4-i) $a>0$ and $h(t) \equiv$ a for $t \in[1, T]_{\mathrm{Z}}$;

(4-ii) $a>0$ and $h(t)>$ a for $t \in[1, T]_{\mathrm{Z}}$;

(4-iii) $a \leq 0$ and $h(t) \equiv 0$ for $t \in[1, T]_{\mathbf{Z}}$;

(4-iv) $a \leq 0$ and $h(t)>0$ for $t \in[1, T]_{\mathrm{z}}$.

(5) If one of the following conditions is satisfied, then problem (1.1) has exactly two positive solutions:

(5-i) $a>0$ and $0<h(t)<a$ for $t \in[1, T]_{\mathbf{Z}}$;

(5-ii) $a>0$ and $h(t) \equiv 0$ for $t \in[1, T]_{\mathbf{Z}}$.

Proof Let $\lambda_{*}=g\left(x_{0}\right)$. We only show the proofs of (2-ii) and (2-iii).

(2-ii) By $g^{\prime}$ is strictly increasing, there exists a unique $x_{1}>x_{0}$ such that $g\left(x_{1}\right)=a$ and $g(x)>$ $a$ for all $x>x_{1}$. Clearly, $u(t) \equiv x_{1}$ is a positive solution of (1.1). Assume that $v(t)$ is another positive solution, then $u$ and $v$ are strictly ordered by Lemma 3.4. If $x_{1} \equiv u(t)<v(t), t \in$ $[1, T]_{\mathrm{Z}}$, then $g(u(t)) \equiv a<g(v(t)), t \in[1, T]_{\mathrm{Z}}$, by the fact that $g(x)$ is strictly increasing on $\left[x_{1},+\infty\right)$. Thus, $\Delta^{2} v(t-1)<0, t \in[1, T]_{\mathrm{Z}}$, which implies $\Delta v(T)<\Delta v(0)$, a contradiction. If $v(t)<u(t) \equiv x_{1}$, then the fact that $g(x)<a$ for $x \in\left(0, x_{1}\right)$ shows that $g(v(t))<g(u(t)) \equiv a$ and hence $\Delta^{2} v(t-1)>0, t \in[1, T]_{\mathrm{Z}}$. It follows that $\Delta v(T)>\Delta v(0)$, a contradiction.

(2-iii) We distinguish two cases to finish the proof.

Case 1: $\lambda_{*}=a=g(0)$. In this case, $g$ is strictly increasing on $[0,+\infty)$. It is easy to see that (1.1) has at least one positive solution by a discussion similar to Theorem 4.1(4-iii). Now, we assume that (1.1) has two distinct positive solutions $u_{1}$ and $u_{2}$. Then $u_{1}$ and $u_{2}$ 
are strictly ordered by Lemma 3.4. Without loss of generality, we assume that $u_{1}(t)<u_{2}(t)$, $t \in[0, T+1]_{\mathbf{Z}}$. Then $g\left(u_{1}(t)\right)<g\left(u_{2}(t)\right), t \in[0, T+1]_{\mathbf{Z}}$, which implies that $\Delta^{2}\left(u_{1}-u_{2}\right)(t-1)=$ $g\left(u_{2}(t)\right)-g\left(u_{1}(t)\right)>0, t \in[1, T]_{\mathbf{Z}}$. Thus, we have the contradiction that

$$
\Delta u_{1}(T)-\Delta u_{2}(T)>\Delta u_{1}(0)-\Delta u_{2}(0)
$$

Case 2: $\lambda_{*}<a$. By $g^{\prime}$ is strictly increasing, there exists a unique $x_{1}>x_{0}$ such that $g\left(x_{1}\right)=a$ and $g(x)>a$ for all $x>x_{1}$. Since $h(t)>a$, there exist $c_{1}$ and $c_{2}: x_{1}<c_{1}<c_{2}$ such that $g\left(c_{1}\right)<$ $h(t), g\left(c_{2}\right)>h(t), t \in[1, T]_{\mathrm{Z}}$. By the proof of Theorem 4.1(4-iii), we know that (1.1) has a positive solution $u_{1}$ with $c_{1} \leq u_{1}(t) \leq c_{2}, t \in[1, T]_{\mathrm{Z}}$. Now, we assume that $u_{2}$ is also a positive solution of (1.1). Then $u_{1}$ and $u_{2}$ are strictly ordered by Lemma 3.4.

If $u_{2}(t)<u_{1}(t), t \in[1, T]_{\mathrm{Z}}$, then $g\left(u_{2}(t)\right)<g\left(u_{1}(t)\right), t \in[1, T]_{\mathrm{Z}}$. In fact, For any given $t$, if $x_{0}<u_{2}(t)<u_{1}(t)$, then $g\left(u_{2}(t)\right)<g\left(u_{1}(t)\right)$ since $g$ is strictly increasing on $\left[x_{0},+\infty\right)$. If $0<u_{2}(t) \leq x_{0}$, then we also have $g\left(u_{2}(t)\right) \leq a=g\left(x_{1}\right)<g\left(c_{1}\right) \leq g\left(u_{1}(t)\right), t \in[1, T]_{\mathrm{Z}}$. Thus, $\Delta^{2}\left(u_{1}-u_{2}\right)(t-1)=g\left(u_{2}(t)\right)-g\left(u_{1}(t)\right)<0, t \in[1, T]_{\mathbf{Z}}$. It follows that $\Delta u_{1}(T)-\Delta u_{2}(T)<$ $\Delta u_{1}(0)-\Delta u_{2}(0)$, a contradiction.

If $u_{2}(t)>u_{1}(t), t \in[1, T]_{\mathrm{Z}}$, then, by the monotony of $g$ on $\left[x_{0},+\infty\right), g\left(u_{1}(t)\right)<g\left(u_{2}(t)\right)$, $t \in[1, T]_{\mathrm{Z}}$, and consequently, $\Delta^{2}\left(u_{1}-u_{2}\right)(t-1)=g\left(u_{2}(t)\right)-g\left(u_{1}(t)\right)>0, t \in[1, T]_{\mathrm{Z}}$, which also yields the contradiction that $\Delta u_{1}(T)-\Delta u_{2}(T)>\Delta u_{1}(0)-\Delta u_{2}(0)$.

The proof is complete.

Finally, we consider the case that $g \in C^{1}(\langle 0,+\infty), \mathbf{R})$ and $g$ is strictly concave on $\langle 0,+\infty)$. If $g \in C^{1}((0,+\infty)$, $\mathbf{R})$, we have the following result by Theorem 3.6 and Theorem 4.4.

Theorem 5.5 Assume $g \in C^{1}((0, \infty), \mathbf{R}), g^{\prime}(x)$ is strictly decreasing and $g^{\prime}(x)<4 \sin ^{2} \frac{\pi}{4 T}$ for all $x \in(0, \infty)$. Let

$$
\lim _{x \rightarrow 0} g(x)=\lim _{x \rightarrow+\infty} g(x)=-\infty, \quad \lambda^{*}=\max _{x \in(0,+\infty)} g(x) \geq 0 .
$$

(1) If $h(t) \geq \lambda^{*}$ and $h(t) \not \equiv \lambda^{*}$ for $t \in[1, T]_{\mathrm{Z}}$, then problem (1.1) has no positive solution.

(2) If $h(t) \equiv \lambda^{*}$ for $t \in[1, T]_{\mathrm{Z}}$, then problem (1.1) has exactly one positive solution.

(3) If one of the following conditions is satisfied, then problem (1.1) has exactly two positive solutions:

(3-i) $0<h(t) \leq \lambda^{*}$ and $h(t) \not \equiv \lambda^{*}$ for $t \in[1, T]_{\mathrm{Z}}$;

(3-ii) $\lambda^{*}>0$ and $h(t) \equiv 0$ for $t \in[1, T]_{\mathrm{z}}$.

If $g \in C^{1}([0,+\infty), \mathbf{R})$, we have the following result.

Theorem 5.6 Assume $g \in C^{1}([0, \infty), \mathbf{R}), g^{\prime}(x)$ is strictly decreasing and $g^{\prime}(x)<4 \sin ^{2} \frac{\pi}{4 T}$ for all $x \in[0, \infty)$. Let

$$
g(0)=a, \quad \lim _{x \rightarrow+\infty} g(x)=-\infty, \quad \lambda^{*}=\max _{x \in[0,+\infty)} g(x)>\max \{0, a\}
$$

(1) If $h(t) \geq \lambda^{*}$ and $h(t) \not \equiv \lambda^{*}$ for $t \in[1, T]_{\mathrm{Z}}$, then problem (1.1) has no positive solution.

(2) If one of the following conditions is satisfied, then problem (1.1) has exactly one positive solution: 
(2-i) $h(t) \equiv \lambda^{*}$ for $t \in[1, T]_{\mathbf{Z}}$;

(2-ii) $a>0$ and $h(t) \equiv a$ for $t \in[1, T]_{\mathrm{Z}}$;

(2-iii) $a>0$ and $0<h(t)<a$ for $t \in[1, T]_{\mathrm{Z}}$

(2-iv) $a \geq 0$ and $h(t) \equiv 0$ for $t \in[1, T]_{\mathrm{z}}$.

(3) If one of the following conditions is satisfied, then problem (1.1) has exactly two positive solutions:

(3-i) $\max \{0, a\}<h(t) \leq \lambda^{*}$ and $h(t) \not \equiv \lambda^{*}$ for $t \in[1, T]_{\mathrm{Z}}$;

(3-ii) $a<0$ and $h(t) \equiv 0$ for $t \in[1, T]_{\mathbf{Z}}$.

\section{Competing interests}

The authors declare that they have no competing interests.

\section{Authors' contributions}

All authors jointly worked on the results and they read and approved the final manuscript.

\section{Author details}

${ }^{1}$ School of Mathematics and Information Science, Guangzhou University, Guangzhou, 510006, P.R. China. ${ }^{2}$ Key Laboratory of Mathematics and Interdisciplinary Sciences of Guangdong Higher Education Institutes, Guangzhou University, Guangzhou, 510006, P.R. China. ${ }^{3}$ School of Science, China University of Geosciences, Beijing, 100083, P.R. China. ${ }^{4}$ Department of Mathematics, Northwest Normal University, Lanzhou, 730070, P.R. China. ${ }^{5}$ Department of Mathematics, North University of China, Taiyuan, Shanxi 030051, P.R. China. ${ }^{6}$ School of Mathematical and Natural Sciences, Arizona State University, Phoenix, AZ 85069-7100, USA.

\section{Acknowledgements}

The authors are very grateful to the anonymous referees for their valuable suggestions. This work was done when Bai was visiting the School of Mathematical and Natural Sciences, Arizona State University. He would like to thank the school for its hospitality. The research is supported partially by the Research Funds for the Doctoral Program of Higher Education of China (No. 20124410110001), by PCSIRT of China (No. IRT1226) and by the Natural Science Fund of China (No. 11371107).

Received: 18 August 2015 Accepted: 21 November 2015 Published online: 08 December 2015

\section{References}

1. Agarwal, RP, Perera, K, O'Regan, D: Multiple positive solutions of singular discrete $p$-Laplacian problems via variational methods. Adv. Differ. Equ. 2005(2), 93-99 (2005)

2. Cabada, A: Extremal solutions for the difference $\phi$-Laplacian problem with nonlinear functional boundary conditions. Comput. Math. Appl. 42, 593-601 (2001)

3. Anderson, D, Avery, RI: Multiple positive solutions to a third-order discrete focal boundary value problem. Comput. Math. Appl. 42, 333-340 (2001)

4. Yu, J, Guo, Z: On generalized discrete boundary value problems of Emden-Fowler equation. Sci. China Ser. A 36 $721-732(2006)$

5. Anderson, DR: Discrete third-order three-point right-focal boundary value problems. Comput. Math. Appl. 45 , 861-871 (2003)

6. Aykut, N: Existence of positive solutions for boundary value problems of second-order functional difference equations. Comput. Math. Appl. 48, 517-527 (2004)

7. Karaca, IY: Discrete third-order three-point boundary value problem. J. Comput. Appl. Math. 205, 458-468 (2007)

8. Yu, J, Guo, Z: On boundary value problems for a discrete generalized Emden-Fowler equation. J. Differ. Equ. 231, 18-31 (2006)

9. Jiang, L, Zhou, Z: Existence of nontrivial solutions for discrete nonlinear two point boundary value problems. Appl. Math. Comput. 180, 318-329 (2006)

10. Yang, Y, Meng, F: Eigenvalue problem for finite difference equations with p-Laplacian. J. Appl. Math. Comput. 40, 319-340 (2012)

11. Ji, D, Ge, W: Existence of multiple positive solutions for Sturm-Liouville-like four-point boundary value problem with p-Laplacian. Nonlinear Anal. 68, 2638-2646 (2008)

12. Liu, Y: Existence results for positive solutions of non-homogeneous BVPs for second order difference equations with one-dimensional p-Laplacian. J. Korean Math. Soc. 47, 135-163 (2010)

13. Bai, $\mathrm{D}, \mathrm{Xu}, \mathrm{X}$ : Existence and multiplicity of difference $\phi$-Laplacian boundary value problems. Adv. Differ. Equ. 2013 $267(2013)$

14. Bai, D: A global result for discrete $\phi$-Laplacian eigenvalue problems. Adv. Differ. Equ. 2013, 264 (2013)

15. Bisci, GM, Repovš, D: On sequences of solutions for discrete anisotropic equations. Expo. Math. 32(3), $284-295$ (2014)

16. Bisci, GM, Repovš, D: Existence of solutions for $p$-Laplacian discrete equations. Appl. Math. Comput. 242, 454-461 (2014)

17. Malin, M: Multiple solutions for a class of oscillatory discrete problems. Adv. Nonlinear Anal. 4(3), 221-233 (2015)

18. Ouyang, T, Shi, J: Exact multiplicity of positive solutions for a class of semilinear problems. J. Differ. Equ. 146(1), $121-156(1998)$

19. Ouyang, T, Shi, J: Exact multiplicity of positive solutions for a class of semilinear problems, II. J. Differ. Equ. 158(1), 94-151 (1999) 
20. Korman, $\mathrm{P}, \mathrm{Li}, \mathrm{Y}$, Ouyang, T: An exact multiplicity result for a class of semilinear equations. Commun. Partial Differ. Equ. 22(3-4), 661-684 (1997)

21. Ortega, R, Zhang, M: Optimal bounds for bifurcation values of a superlinear periodic problem. Proc. R. Soc. Edinb. A 35, 119-132 (2005)

22. Chen, H, Li, Y: Stability and exact multiplicity of periodic solutions of Duffing equations with cubic nonlinearities. Proc. Am. Math. Soc. 135, 3925-3932 (2007)

23. Lee, E, Sasi, S, Shivaji, R: S-shaped bifurcation curves in ecosystems. J. Math. Anal. Appl. 381, 732-741 (2011)

24. Simon, PL: Exact multiplicity of positive solutions for a class of singular semilinear equations. Differ. Equ. Dyn. Syst. 18(4), 415-429 (2010)

25. Pan, $\mathrm{H}$, Xing, R: Time maps and exact multiplicity results for one-dimensional prescribed mean curvature equations. Nonlinear Anal. 74, 1234-1260 (2011)

26. Tzeng, C, Hung, K, Wang, S: Global bifurcation and exact multiplicity of positive solutions for a positone problem with cubic nonlinearity. J. Differ. Equ. 252, 6250-6274 (2012)

27. Feng, Y: Sobolev inequality and the exact multiplicity of solutions and positive solutions to a second-order Neumann boundary value problem. Acta Appl. Math. 110, 895-905 (2010)

28. Rădulescu, V: Nonlinear elliptic equations with variable exponent: old and new. Nonlinear Anal. TMA 121, 336-369 (2015)

29. Rădulescu, V, Repovš, D: Partial Differential Equations with Variable Exponents: Variational Methods and Qualitative Analysis. CRC Press, Boca Raton (2015)

30. Walter, KK, Allan, CP: Difference Equations. Academic Press, San Diego (1991)

31. Amann, H: Fixed point equations and nonlinear eigenvalue problems in ordered Banach spaces. SIAM Rev. 18, 620-709 (1976)

\section{Submit your manuscript to a SpringerOpen ${ }^{\circ}$ journal and benefit from:}

- Convenient online submission

- Rigorous peer review

Immediate publication on acceptance

- Open access: articles freely available online

- High visibility within the field

- Retaining the copyright to your article 